\title{
ALPHA : Personal Assistant in Chat Box
}

\author{
Shubham Jain ${ }^{1}$, Shreya Joshi ${ }^{1}$, Ruchi Parashar ${ }^{2}$ \\ ${ }^{1}$ Dr. Akhilesh Das Gupta Institute of Technology and Management New Delhi, Department of Computer \\ Science and Engineering, GGSIPU, New Delhi, India \\ ${ }^{2}$ Assistant Professor, Dr. Akhilesh Das Gupta Institute of Technology and Management New Delhi, \\ Department of Computer Science and Engineering, GGSIPU, New Delhi, India
}

\begin{abstract}
Article Info

Volume 7, Issue 6

Page Number : 309-312

Publication Issue :

November-December-2021

\section{Article History}

Accepted : 05 Dec 2021

Published : 18 Dec 2021

This research paper gives a comprehensive view of a virtual assistant named "Alpha" which is developed using the concept of Artificial intelligence, to aid in education, market, business and many other fields.This bot is programmed in python and is stored in raspberry pi, providing it a user friendly environment by making the bot move along with the user. A virtual assistant is an application that takes voice input, processes it and then gives output according to the input. Alpha is a real time, interactive bot and it is built on the latest technologies. It uses numerous python Libraries to help perform various functions that enable the Assistant to assist its user in day to day activities. The Assistant can convert text to speech and vice versa using gTTS and speech Recognition libraries respectively. It is a multilingual digital employee that speaks, listens and comprehends over 26 languages. It has other interesting features of face recognition and registration, smog sensing and alcohol sensing. It is also capable of making payments using the QR scanner feature added to it.

Keywords : Python, Artificial intelligence, the Gmail sender, Internet of things
\end{abstract}

\section{INTRODUCTION}

A virtual assistant or virtual employee assistant is an application that takes natural language voice as input commands to perform tasks for the user. It takes voice input, processes it and finally gives output according to the input. The virtual Assistant is based on python and uses emerging technologies like voice recognition, face recognition and AI. Unlike other assistants, this virtual assistant can also make different sorts of lists like a shopping list, to-do list etc. On the user's voice command, it can perform multiple tasks like play music, open any website, open YouTube, surf YouTube, open some games, open any sort of presaved file from your desktop, send Gmail and much more.

The specialty of this Assistant is that it is loaded in a hardware called Raspberry pi. Along with pi, it contains some more hardware devices such as ARDUINO, fire sensor, smoke sensor, buzzer, etc. This assistant, by sensing the colour can start or stop 
moving and it can move along with the user. With all these features it comes with a smoke sensor which can sense smoke within 3 meters, ring an alarm and after the alarm, it can find a way to exit in order to protect itself. It can also detect whether a person is drunk and hence fit for driving or not.It is a multilingual digital employee that speaks, listens and comprehends over 26 languages.

It is also capable of making payments by scanning the QR Code.

This Assistant also comes with interesting games like:

1. Tic-tac-toe: a game made in simple python GUI

2. Flappy birds: a game made with the use of python turtle without the use of GUI

3. Hungry Snake: a snake game with GUI

\section{LITERATURE SURVEY}

A number of virtual assistants are available these days. Some of them are:

Amazon's Alexa, Microsoft's Cortana, Apple’s Siri. Siri is a personal assistant that uses natural language processing to answer questions and outsource requests to web services that will then be carried out for the user. [6]

Let's talk about Google's Assistant because it is selfsufficient and covers almost every spectrum of assistance.[2]

The Google Assistant offers voice commands, voice searching, and voice-activated device control which lets you to complete a number of tasks after you've prompted "OK Google" or "Hey, Google" wake words. The assistant is designed to give you conversational interactions. It can control your devices and your smartphone via voice. It has the ability to access your personal information such as contact numbers, calendar dates, events, bookings, etc. It can also read the weather for you and inform you if the route you are travelling on is jammed from an accident or not. The Google Assistant also comes with the feature of setting alarms and reminders using simple voice commands. It is capable of running timers, sending messages, recording your voice and even singing for you.

\section{IMPLEMENTATION}

The implementation of our virtual assistant is given through the following diagrams

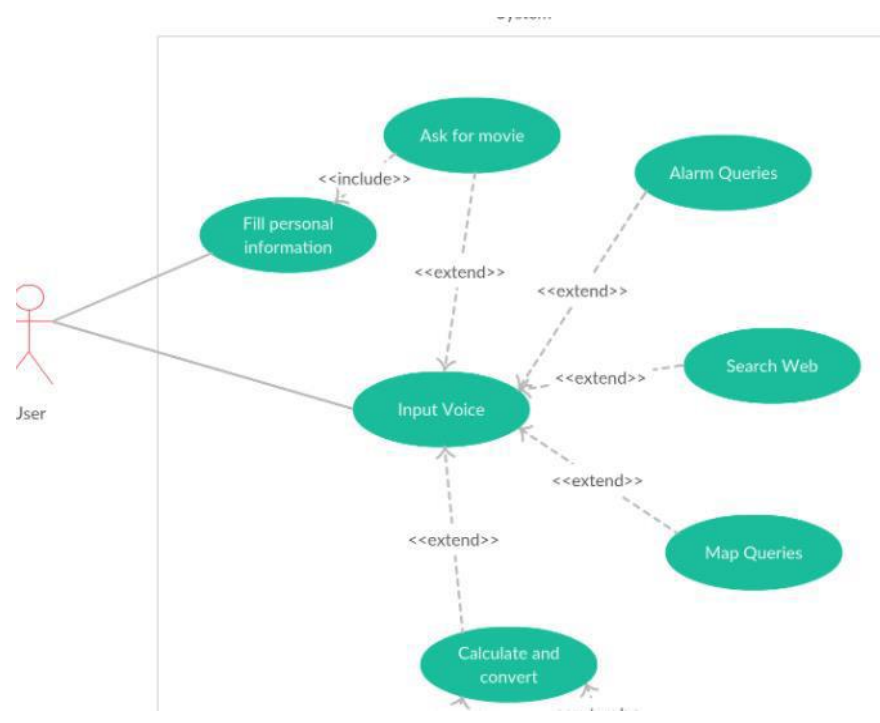

Figure 1: Outline of Software

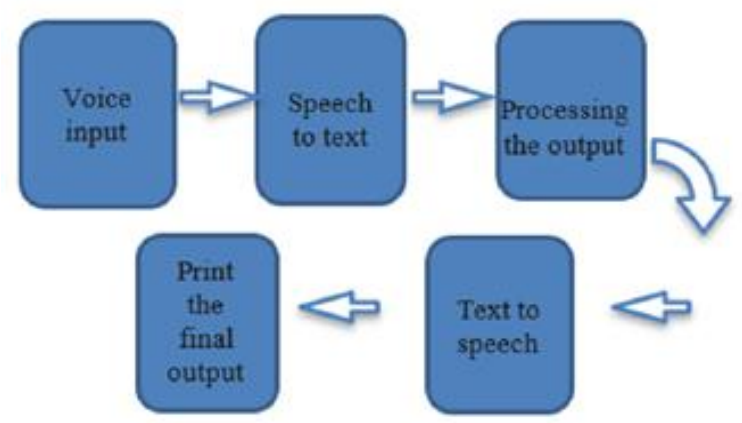

Figure 2: Working of Speech to Text and Text to

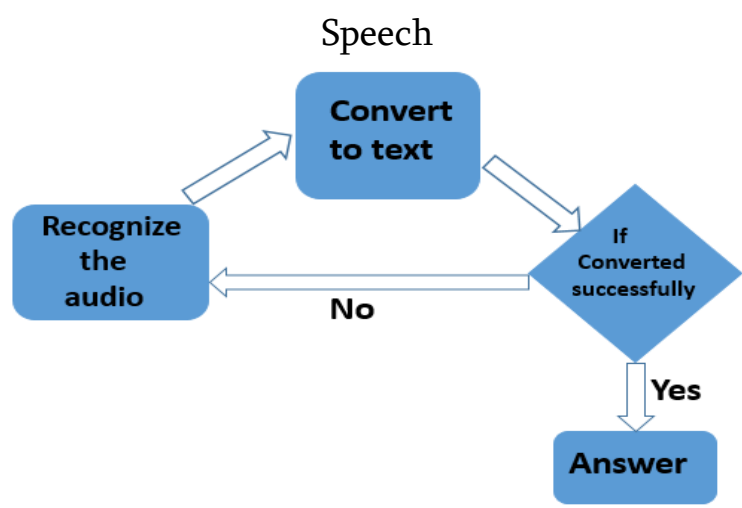

Figure 3: Correction Mechanism of Figure 2 


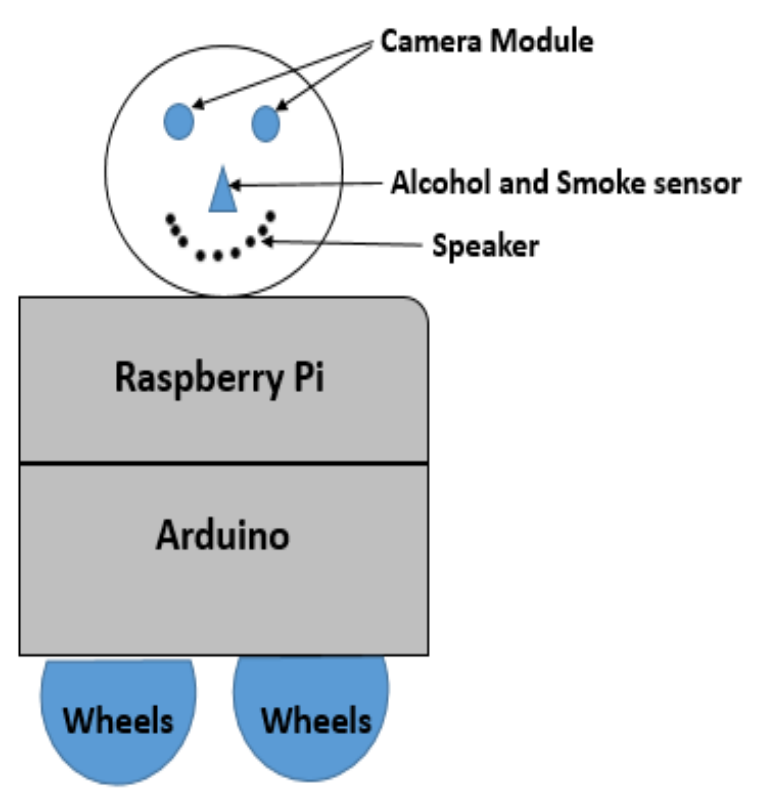

Figure 4: Hardware Outline

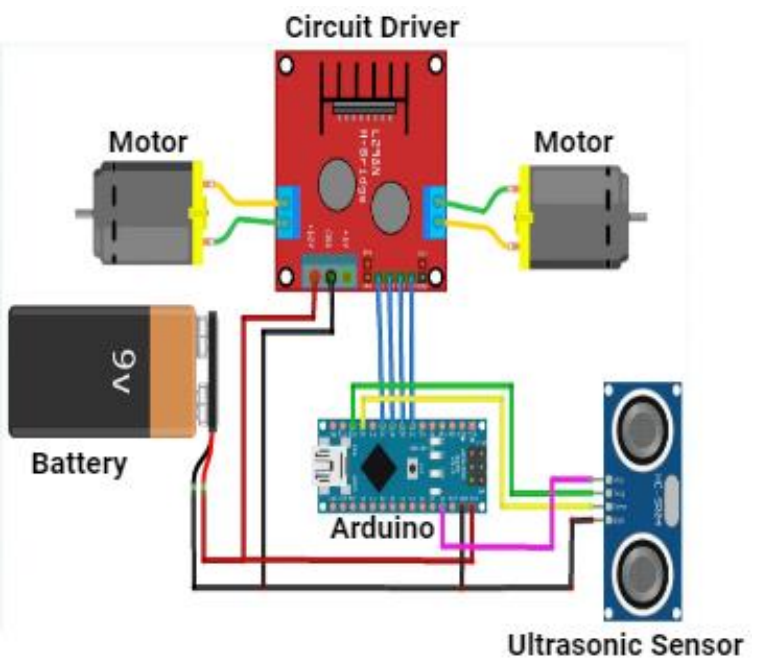

Figure 5: Circuit Diagram

The software requirements for this virtual assistant are as follows:

\section{Anaconda 3.0}

2. A system with windows 10 or above OR raspbian OS

Hardware Requirements are as follows:

1. Camera module

2. Alcohol sensor

3. Smog sensor

4. USB mic

5. Speaker (aux type)
6. Raspberry Pi

7. ARDUINO

\section{RESULT}

This AI-powered Bot can recognize speech, comprehend text, and scan the data to answer your queries in nearly the way we humans do. It uses speech recognition, and text recognition features to answer your queries. This AI assistant is trained first with examples and once this is done it starts recording the inputs, doing the processing, and producing the desired results.

So, we can call ALPHA a success.

\section{FUTURE SCOPE}

1. Recommendation System: to suggest relevant items to the user

2. SOS feature: emergency contact to the guardian of the user if he/she is in danger

3. Some other biometrics such as fingerprint scanner, Iris scanner can also be attached with it.

4. Since we are working on raspberry pi, pulse monitoring and blood sugar monitoring sensors can also be added

5. Advance use of facial recognition such that it is able to detect the current mood of the user by studying his facial expressions.[4]

6. Increase its efficiency by adding a feature through which it can store the past activities of the user in a specific region and then use that data to make automatic reminders for the tasks that are performed daily.[2]

\section{REFERENCES}

[1]. Chatani M, How AI Is Empowering Rakuten Customers Today (And How It Might Help Them Tomorrow). Rakuten Today. 15/12/2018 https://rakuten.today/tag/ai . 
[2]. Columbus L, Roundup of Machine Learning Forecasts and Market Estimates, Forbes, $18 / 02 / 2018$

https://www.forbes.com/sites/louiscolumbus/

[3]. Foye L Chatbot Conversations to deliver $\$ 8$ billion in Cost savings by 2022. Juniper Research. $24 \quad$ July 2018 https://www.juniperresearch.com/analystxpress

[4]. Frank Aaron Machines Teaching Each Other Could Be the Biggest Exponential Trend in AI. Singularity hub. 15/10/2018 https://singularityhub.com/frankaaron

[5]. Learn Python the Hard Way' by Zed A. Shaw 'Python Cook Book' by David Beazley and Brian Jones

[6]. https://lup.lub.lu.se/record/4317727

\section{Cite this article as :}

Shubham Jain, Shreya Joshi, Ruchi Parashar, "ALPHA : Personal Assistant in Chat Box", International Journal of Scientific Research in Computer Science, Engineering and Information Technology (IJSRCSEIT), ISSN : 2456-3307, Volume 7 Issue 6, pp. 309-312, November-December 2021. Available at

doi : https://doi.org/10.32628/CSEIT217643

Journal URL : https://ijsrcseit.com/CSEIT217643 